\title{
Endogenous Endothelins Mediate Increased Distal Tubule Acidification Induced by Dietary Acid in Rats
}

\author{
Donald E. Wesson \\ Departments of Internal Medicine and Physiology, Texas Tech University Health Sciences Center, Lubbock, Texas 79430
}

\begin{abstract}
We examined if endogenous endothelins mediate the decreased $\mathrm{HCO}_{3}$ secretion and increased $\mathrm{H}^{+}$secretion in in vivo-perfused distal tubules of rats fed dietary acid as $\left(\mathrm{NH}_{4}\right)_{2} \mathrm{SO}_{4}$. Animals given $\left(\mathrm{NH}_{4}\right)_{2} \mathrm{SO}_{4}$ drinking solution had higher endothelin-1 addition to renal interstitial fluid than those given distilled $\mathrm{H}_{2} \mathrm{O}(480 \pm 51$ vs. $293 \pm 32 \mathrm{fmol} \mathrm{g}$ kidney $\mathrm{wt}^{-1} \mathrm{~min}^{-}$, respectively, $\left.P<0.03\right) .\left(\mathrm{NH}_{4}\right)_{2} \mathrm{SO}_{4}$-ingesting animals infused with bosentan $(10 \mathrm{mg} / \mathrm{kg})$ to inhibit $\mathrm{A}-$ and B-type endothelin receptors had higher $\mathrm{HCO}_{3}$ secretion than baseline $\left(\mathrm{NH}_{4}\right)_{2} \mathrm{SO}_{4}$ animals $(-4.7 \pm 0.4$ vs. $-2.4 \pm 0.3 \mathrm{pmol}$ $\left.\mathrm{mm}^{-1} \min ^{-1}, P<0.01\right)$, but $\left(\mathrm{NH}_{4}\right)_{2} \mathrm{SO}_{4}$ animals given a specific inhibitor of A-type endothelin receptors (BQ-123) did not $\left(-2.0 \pm 0.2 \mathrm{pmol} \mathrm{mm}^{-1} \min ^{-1}, P=\mathrm{NS}\right.$ vs. baseline $) . \mathrm{H}^{+}$ secretion was lower in bosentan-infused compared with baseline $\left(\mathrm{NH}_{4}\right)_{2} \mathrm{SO}_{4}$ animals $(27.7 \pm 2.5$ vs. $43.9 \pm 4.0$ pmol $\left.\mathrm{mm}^{-1} \mathrm{~min}^{-1}, P<0.03\right)$, but that for BQ-123-infused $\left(\mathrm{NH}_{4}\right)_{2} \mathrm{SO}_{4}$ animals was not $\left(42.9 \pm 4.2 \mathrm{pmol} \mathrm{mm} \mathrm{mm}^{-1} \mathrm{~min}^{-1}\right.$, $\boldsymbol{P}=$ NS vs. baseline). Bosentan had no effect on distal tubule $\mathrm{HCO}_{3}$ or $\mathrm{H}^{+}$secretion in control animals. The data show that dietary acid increases endothelin-1 addition to renal interstitial fluid and that inhibition of B- but not A-type endothelin receptors blunts the decreased $\mathrm{HCO}_{3}$ secretion and increased $\mathrm{H}^{+}$secretion in the distal tubule of animals given dietary acid. The data are consistent with endogenous endothelins as mediators of increased distal tubule acidification induced by dietary acid. (J. Clin. Invest. 1997. 99: 2203-2211.) Key words: bicarbonate - bosentan • interstitium $\bullet$ micropuncture $\cdot$ proton $\bullet$ secretion
\end{abstract}

\section{Introduction}

Dietary acid increases acidification in the distal tubule in vivo (1-3), but the factors that induce this response are unknown. This dietary maneuver might increase distal tubule acidification directly through changes in acid-base parameters of body fluids and/or indirectly by altering production of substances that modulate distal tubule acidification. In previous studies from this laboratory, animals ingesting the acid-producing salt $\left(\mathrm{NH}_{4}\right)_{2} \mathrm{SO}_{4}$ increased distal tubule acidification and urine acid

Address correspondence to Donald E. Wesson, Texas Tech University Health Sciences Center, Renal Section, 3601 Fourth St., Lubbock, TX 79430. Phone: 806-743-3155; FAX: 806-743-3148; E-mail: phydew@ttuhsc.edu

Received for publication 20 September 1996 and accepted in revised form 20 February 1997.

J. Clin. Invest.

(C) The American Society for Clinical Investigation, Inc.

0021-9738/97/05/2203/09 \$2.00

Volume 99, Number 9, May 1997, 2203-2211 excretion without sustained changes in plasma acid-base parameters (3). Furthermore, intracellular $\mathrm{pH}$ of cultured renal epithelial cells chronically ( $48 \mathrm{~h})$ exposed to acid media was not different from control, yet these cells had increased $\mathrm{Na}^{+} / \mathrm{H}^{+}$ antiporter activity (4). These data suggest that persistent alterations in plasma or intracellular fluid acid-base parameters are not necessary to permit a sustained increase in renal epithelial acidification. Thus, dietary acid might increase distal nephron acidification indirectly by altering production of substances that more directly influence the components of distal tubule acidification. The mechanism by which the distal tubule increases acidification in response to dietary acid might suggest substances responsible for inducing the change. Our previous studies showed that dietary acid decreases $\mathrm{HCO}_{3}$ secretion and increases $\mathrm{H}^{+}$-secreting capacity in distal tubules in vivo (3). Endothelin-1 (ET-1), ${ }^{1}$ an agent made by collecting tubules (5) and renal microvascular endothelium (6), decreases distal tubule $\mathrm{HCO}_{3}$ secretion induced by dietary $\mathrm{HCO}_{3}$ (7) and increases $\mathrm{Na}^{+} / \mathrm{H}^{+}$exchanger (NHE)-3 activity in cultured renal epithelia (8). The data show that directional changes in components of distal tubule acidification induced by dietary acid are consistent with ET-1 actions on tubule $\mathrm{HCO}_{3} / \mathrm{H}^{+}$secretion.

The present studies used free-flow and in vivo microperfusion micropuncture to test the hypothesis that augmented endothelin secretion mediates increased distal tubule acidification induced by dietary acid. The data show that dietary acid increases ET-1 addition to renal interstitial fluid, a fluid with access to both the renal epithelium and endothelium. Furthermore, the studies show that endothelin receptor inhibition blunts both the decrease in distal tubule $\mathrm{HCO}_{3}$ secretion and increase in $\mathrm{H}^{+}$-secreting capacity induced by dietary acid. The data support the hypothesis that endothelin mediates the increase in distal tubule acidification induced by dietary acid.

\section{Methods}

Male and female Munich-Wistar rats (Harlan Sprague-Dawley Co., Houston, TX) weighing 240-271 g were used. Previous studies showed that dietary $\left(\mathrm{NH}_{4}\right)_{2} \mathrm{SO}_{4}$ decreased $\mathrm{HCO}_{3}$ secretion and increased $\mathrm{H}^{+}$secreting capacity in the rat distal tubule in vivo (3). We used the same $\left(\mathrm{NH}_{4}\right)_{2} \mathrm{SO}_{4}$-ingesting protocol in the present studies and these animals will be referred to as acid ingesting. Experimental and control animals eating a minimum electrolyte diet (ICN Nutritional Biochemicals, Cleveland, $\mathrm{OH})$ received $40 \mathrm{mM}\left(\mathrm{NH}_{4}\right)_{2} \mathrm{SO}_{4}$ drinking solution and distilled $\mathrm{H}_{2} \mathrm{O}$, respectively, for 7-10 d before micropuncture. Because $\left(\mathrm{NH}_{4}\right)_{2} \mathrm{SO}_{4}$-ingesting animals had higher urine flow than control and because diuresis might increase urine ET-1 excretion (9), we studied a second control group ingesting $40 \mathrm{mM}$ $\mathrm{Na}_{2} \mathrm{SO}_{4}$ drinking solution that had similar drinking volumes and urine flows to $\left(\mathrm{NH}_{4}\right)_{2} \mathrm{SO}_{4}$ animals. Bosentan (Hoffman-LaRoche, Basel,

1. Abbreviations used in this paper: ET-1, endothelin-1; $\mathrm{J}_{\mathrm{V}}$, fluid reabsorption; NAE, net acid excretion; NHE, $\mathrm{Na}^{+} / \mathrm{H}^{+}$exchanger; RIF, renal interstitial fluid. 
Switzerland), a nonpeptide endothelin ${ }_{\mathrm{A}}$ and endothelin $\mathrm{B}_{\mathrm{B}}$ receptor antagonist (10), was infused (10 mg/kg i.v.) to antagonize actions of endogenous endothelins. This bosentan dose inhibits initial depressor and sustained pressor responses to ET-1 $>60 \%$ as long as $6 \mathrm{~h}$ (10). To distinguish endothelin ${ }_{\mathrm{A}}$ - from endothelin $\mathrm{B}_{\mathrm{B}}$-mediated effects, we infused a comparison animal group with BQ-123 (Bachem California, Torrence, $\mathrm{CA}$ ), a selective endothelin ${ }_{\mathrm{A}}$ receptor antagonist (11), $1 \mathrm{mg} / \mathrm{kg}$ i.v. followed by $0.1 \mathrm{mg} / \mathrm{kg}$ per h. This BQ-123 dose inhibited pressor responses to both stimulated endogenous endothelins and infused ET-1 (12).

Urine net acid excretion. Daily net acid excretion (NAE) was measured as described (13) in four each of control, $\left(\mathrm{NH}_{4}\right)_{2} \mathrm{SO}_{4}$, and $\mathrm{Na}_{2} \mathrm{SO}_{4}$ animals fed drinking solution and diet as described. Because rats of similar weight ingested $15.6 \pm 0.6 \mathrm{~g} / \mathrm{d}$ of diet when drinking distilled $\mathrm{H}_{2} \mathrm{O}, 16.7 \pm 0.8 \mathrm{~g} / \mathrm{d}$ for the $\left(\mathrm{NH}_{4}\right)_{2} \mathrm{SO}_{4}$ solution, and $15.9 \pm 0.6 \mathrm{~g} / \mathrm{d}$ for the $\mathrm{Na}_{2} \mathrm{SO}_{4}$ solution, each animal was fed exactly $15 \mathrm{~g} / \mathrm{d}$ of diet to ensure that each group ingested the same amount. The diet contained $20 \%$ protein and the following electrolytes (in $\mu \mathrm{eq} / \mathrm{g}$ diet): $21.7 \mathrm{Na}^{+}$, $43.5 \mathrm{~K}^{+}$, and $13.8 \mathrm{Cl}^{-}$. All groups drank distilled $\mathrm{H}_{2} \mathrm{O}$ with diet for $48 \mathrm{~h}$, and then were given either $40 \mathrm{mM}\left(\mathrm{NH}_{4}\right)_{2} \mathrm{SO}_{4}, 40 \mathrm{mM} \mathrm{Na}_{2} \mathrm{SO}_{4}$, or distilled $\mathrm{H}_{2} \mathrm{O}$ (control) for an additional $7 \mathrm{~d}$ while in metabolic cages. On each of the subsequent $7 \mathrm{~d}$, animals were anesthetized with ketamine $\mathrm{HCl}(100 \mathrm{mg} / \mathrm{kg}$ body wt; Parke-Davis, Morris Plains, NJ) and bladder urine anaerobically obtained by percutaneous puncture with a $25-$ gauge needle as done previously (13) for NAE measurement. After anesthesia recovery and excreting blood-free urine, they returned to metabolic cages to continue their drinking solutions. We examined NAE in an additional set of bosentan-infused compared with vehicleinfused animals from each of the three experimental groups after they had ingested their drinking solutions and diet for $7 \mathrm{~d}$. On the evening of the 7th day, animals were anesthetized as described and 10 $\mathrm{mg} / \mathrm{kg}$ bosentan or vehicle infused into the jugular vein. The wound was closed with surgical clips and NAE was measured as described for the next $12 \mathrm{~h}$.

Microdialysis technique for measurement of renal interstitial fluid ET-1. Renal interstitial fluid (RIF) ET-1 addition was estimated using microdialysis of the renal cortical interstitium (14). A microdialysis apparatus was constructed from a 5-mm-long piece of hollow fiber dialysis tubing (molecular mass cutoff 5,000 D; Hospal, Meyzieu, France) with $0.1-\mathrm{mm}$ inner diameter as described (14). Each end of the dialysis tubing was connected to a $25-\mathrm{cm}$-long polyethylene tube (0.12-mm inner diameter, 0.65 -mm outer diameter; Bioanalytical systems, Indianapolis, IN) and sealed in place with cyanoacrylic glue (14). The left kidney was exposed through a flank incision in anesthetized rats and its renal capsule penetrated with a 31-gauge needle that was tunneled in the outer renal cortex $\sim 1 \mathrm{~mm}$ from the renal surface for $\sim 0.5 \mathrm{~mm}$ before exiting by penetrating the renal capsule again. The tip of the needle was inserted into one end of the dialysis probe and pulled together with the dialysis tube until the dialysis fiber was situated within the renal cortex. The flank wound was closed with clips and the inflow tube connected to a gas-tight syringe filled with lactated Ringer's infused at $3 \mu \mathrm{l} / \mathrm{min}$ (Harvard Apparatus, Inc., South Natick, MA), as done by others (14). To quantitate possible contamination of RIF with tubule contents caused by insertion of the microdialysis apparatus, we infused five animals having the microdialysis apparatus in place with a large amount $(1 \mathrm{mCi})$ of ${ }^{3} \mathrm{H}$-inulin and compared late proximal tubule ${ }^{3} \mathrm{H}$-inulin concentration with that in $\mathrm{RIF}$ as determined by microdialysis. In vitro ${ }^{3} \mathrm{H}$-inulin recovery, evaluated by immersing dialysis membranes of five identically constructed probes into a beaker containing ${ }^{3} \mathrm{H}$-inulin, was $89 \%$. RIF ${ }^{3} \mathrm{H}$-inulin concentration was $4.7 \%$ of that in the late proximal tubule, consistent with minimal leakage of tubule contents into RIF. Three consecutive 20-min collection periods were done in four each of control, $\left(\mathrm{NH}_{4}\right)_{2} \mathrm{SO}_{4}$, and $\mathrm{Na}_{2} \mathrm{SO}_{4}$ animals for RIF ET-1 measurements. In vitro ET-1 recoveries for four identically constructed probes in a beaker with ${ }^{125}$ I-ET-1 (ICM Biomedicals, Irvine, CA) perfused as described was $59 \pm 2 \%$. ET- 1 in RIF dialysate was measured using a RIA kit (Peninsula Laboratories Inc., Belmont, CA) after disposable column extraction (Sep-Pak C18; Millipore Corp., Milford, MA) preconditioned with methanol, $\mathrm{H}_{2} \mathrm{O}$, and acetic acid as described (15).

Micropuncture protocol. Animals were prepared for micropuncture of accessible distal tubules as described (16). This distal nephron segment is comprised of multiple epithelia (17) but we will hereafter refer to it as the "distal tubule" for simplicity. Distal tubules were perfused at the early distal flow rate measured in situ (6 nl/min) (18), calibrated in vitro, and verified in vivo (16). Transepithelial potential difference was measured after perfusate collection for each solution (16). An injected latex cast determined perfused tubule length after subsequent acid digestion of the kidney (16). Anaerobically obtained arterial $(0.35 \mathrm{ml})$ and stellate vessel blood plasma (16) was analyzed for $\mathrm{tCO}_{2}$ using flow-through fluorometry (see below) (19), and for $\mathrm{pH}, \mathrm{PCO}_{2}$, and electrolytes (16). Diet, but not drinking solution, was withheld the evening before studying micropunctured animals, yielding higher baseline $\mathrm{HCO}_{3}$ reabsorption (20) and permitting differences in $\mathrm{HCO}_{3}$ reabsorption to be more clearly seen.

Table I depicts perfusate composition. Standard perfusate $\mathrm{HCO}_{3}$ and $\mathrm{Cl}^{-}$were 5 and $40 \mathrm{mM}$, respectively, to approximate these anion concentrations in early distal tubule fluid of control animals (18). Solution 1 contained no $\mathrm{HCO}_{3}$ to assess blood-to-lumen $\mathrm{HCO}_{3}$ accumulation and to calculate an apparent transtubule $\mathrm{HCO}_{3}$ permeability as done previously (16) and described below. Solution 2 was $\mathrm{HCO}_{3}$ - and $\mathrm{Cl}^{-}$-free and contained $0.5 \mathrm{mM}$ acetazolamide. Acetazolamide inhibits $\mathrm{HCO}_{3}(16,21)$ and $\mathrm{H}^{+}$secretion $(22)$ in the in vivo-perfused rat distal tubule. Thus, measuring luminal $\mathrm{HCO}_{3}$ accumulation and voltage when perfusing with a zero $\mathrm{HCO}_{3}$, zero $\mathrm{Cl}^{-}$, and acetazolamidecontaining solution allows calculation of passive blood-to-lumen transepithelial $\mathrm{HCO}_{3}$ permeability as done in our laboratory (16) and by others (23). Solution 2 was used in this way. Solution 3 contained $5 \mathrm{mM}$ $\mathrm{HCO}_{3}$ for measurement of net $\mathrm{HCO}_{3}$ reabsorption and for calculation of luminal $\mathrm{H}^{+}$secretion using the apparent transtubule $\mathrm{HCO}_{3}$ permeability derived from perfusing with solution 1 as described (16). Solution 4 was identical to solution 3 except that it contained 10 rather than $5 \mathrm{mM} \mathrm{NaHCO}$. This perfusing solution helped to determine if a minimum attainable $\mathrm{HCO}_{3}$ in distal tubule fluid limited net $\mathrm{HCO}_{3}$ reabsorption in the $\left(\mathrm{NH}_{4}\right)_{2} \mathrm{SO}_{4}$-ingesting animals as previously reported from this laboratory (3). All perfusing solutions contained raffinose to minimize fluid transport and permit more focused study of $\mathrm{HCO}_{3}$ transport (16). Three selected distal tubules were perfused in each animal of each group. One distal tubule was perfused with solution 1 , another with solution 2 , and the third was perfused in paired fashion (16) with solutions 3 and 4 in random order. The order of perfusing solutions was random.

Analytical methods. Immediately after experiment termination, initial and collected perfusate, as well as stellate vessel plasma samples, were analyzed for inulin as done previously (16) and for $\mathrm{tCO}_{2}$ using flow-through ultrafluorometry (19) as done previously (24). All tubule fluid and plasma $\mathrm{tCO}_{2}$ were measured on the experimental

Table I. Perfusate Composition in Millimoles

\begin{tabular}{lrrrr}
\hline & \multicolumn{4}{c}{ Solution } \\
\cline { 2 - 5 } & 1 & 2 & 3 & 4 \\
\hline $\mathrm{Na}^{+}$ & 61 & 61 & 61 & 61 \\
$\mathrm{~K}^{+}$ & 4 & 4 & 4 & 4 \\
$\mathrm{Cl}^{-}$ & 40 & 0 & 40 & 40 \\
$\mathrm{HCO}_{3}$ & 0 & 0 & 5 & 10 \\
Gluconate $_{\text {Acetazolamide }}$ & 25 & 65 & 20 & 15 \\
Raffinose & 0 & 0.5 & 0 & 0 \\
& 200 & 200 & 200 & 200
\end{tabular}

All solutions contained $0.5 \% \mathrm{FD} \& \mathrm{C}$ green dye and were equilibrated with $6.7 \% \mathrm{CO}_{2}$. 
day by comparing fluorescence of a 7-8-nl sample aliquot (corrected for a distilled $\mathrm{H}_{2} \mathrm{O}$ blank run with each sample group) to a standard curve as previously described (24). This technique actually measures $\mathrm{tCO}_{2}$, but we will refer to this measured value as $\mathrm{HCO}_{3}$ for simplicity.

Calculations. Net $\mathrm{HCO}_{3}$ transport was the difference between perfused and collected rates. Net $\mathrm{HCO}_{3}$ reabsorption refers to net $\mathrm{HCO}_{3}$ transport obtained when perfusing with initially $\mathrm{HCO}_{3}$-containing solutions. Luminal $\mathrm{HCO}_{3}$ accumulation describes net $\mathrm{HCO}_{3}$ transport obtained when perfusing with initially $\mathrm{HCO}_{3}$-free solutions. A positive value for $\mathrm{HCO}_{3}$ transport indicates net $\mathrm{HCO}_{3}$ movement out of the lumen (reabsorption) and a negative one indicates net $\mathrm{HCO}_{3}$ movement into the lumen (secretion). A transtubule $\mathrm{HCO}_{3}$ permeability $\left(\mathrm{P}_{\mathrm{HCO} 3}\right)$ was calculated as done previously (16). The term "passive" $\mathrm{HCO}_{3}$ permeability refers to that obtained when perfusing with zero $\mathrm{HCO}_{3}$, zero $\mathrm{Cl}^{-}$, and acetazolamide-containing solution (solution 2). By contrast, the term "apparent" permeability refers to transtubule $\mathrm{HCO}_{3}$ permeability obtained when perfusing with zero $\mathrm{HCO}_{3}$, zero $\mathrm{Cl}^{-}$solution that contained no acetazolamide (solution 1). Bicarbonate secretion was estimated during perfusion with $\mathrm{HCO}_{3}$-containing solutions by calculating $\mathrm{HCO}_{3}$ transport into the lumen using the apparent permeability derived from perfusing with the $\mathrm{HCO}_{3}$ free solution (16). Thus, $\mathrm{HCO}_{3}$ secretion = apparent permeability $\times$ transepithelial $\mathrm{HCO}_{3}$ gradient corrected for transepithelial potential difference (16). The same apparent permeability (derived perfusing with solution 1 as discussed) was used to calculate $\mathrm{HCO}_{3}$ secretion when perfusing with both the $5 \mathrm{mM}$ (solution 3) and $10 \mathrm{mM}$ (solution 4) $\mathrm{HCO}_{3}$-containing solutions. By contrast, a unique transepithelial $\mathrm{HCO}_{3}$ gradient was calculated for each $\mathrm{HCO}_{3}$-containing solution using respective tubule $\mathrm{HCO}_{3}$ concentrations measured when perfusing with that solution (16). The transepithelial potential difference used was the one measured when perfusing with the respective $\mathrm{HCO}_{3}$-containing solution. $\mathrm{H}^{+}$secretion was estimated during perfusion with the $\mathrm{HCO}_{3}$-containing perfusates by subtracting calculated $\mathrm{HCO}_{3}$ secretion from measured net $\mathrm{HCO}_{3}$ reabsorption (16). This method for quantifying $\mathrm{H}^{+}$secretion assumes that all $\mathrm{HCO}_{3}$ transport from the lumen (absolute $\mathrm{HCO}_{3}$ reabsorption) is mediated by luminal $\mathrm{H}^{+}$secretion (22). Furthermore, this method underestimates $\mathrm{H}^{+}$secretion to the extent that $\mathrm{HCO}_{3}$ entering the perfusing solution during perfusion is subsequently reabsorbed. The perfusing solutions contained no $\mathrm{NH}_{3} / \mathrm{NH}_{4}{ }^{+}$for reasons previously discussed (25). Fluid reabsorption $\left(\mathrm{J}_{\mathrm{v}}\right)$ was the difference between perfused and collected flow rates. All transport values were corrected for perfused tubule length (in $\mathrm{mm}$ ).

Statistical analysis. The number comprising the three experimental groups represents animals and not tubules. Each reported animal had at least one successful distal tubule perfusion with each perfusing solution. The Bonferroni method was used for $t$ test comparison of means $(P<0.05)$ when multiple different comparisons of the same parameter were done in animals among the three animal groups.

\section{Results}

General response to dietary protocols. There were no body weight differences between $\mathrm{H}_{2} \mathrm{O}$ (control), $\left(\mathrm{NH}_{4}\right)_{2} \mathrm{SO}_{4}$, and $\mathrm{Na}_{2} \mathrm{SO}_{4}$ animals at the start $(255 \pm 8,254 \pm 6$, and $244 \pm 6 \mathrm{~g}$, respectively) or end (268 $\pm 9,263 \pm 6$, and $257 \pm 6 \mathrm{~g}$, respectively) of the experimental period. Food intake was not different among groups. Ingested drinking solution volume was higher in $\left(\mathrm{NH}_{4}\right)_{2} \mathrm{SO}_{4}(37.1 \pm 2.9 \mathrm{ml} / \mathrm{d})$ and $\mathrm{Na}_{2} \mathrm{SO}_{4}(33.2 \pm 2.0 \mathrm{ml} / \mathrm{d})$ animals compared with control $(21.3 \pm 1.8 \mathrm{ml} / \mathrm{d}, P<0.02$ vs. $\left(\mathrm{NH}_{4}\right)_{2} \mathrm{SO}_{4}$ and $\mathrm{Na}_{2} \mathrm{SO}_{4}$ groups). Similarly, urine flow was higher in $\left(\mathrm{NH}_{4}\right)_{2} \mathrm{SO}_{4}(21.1 \pm 1.9 \mathrm{ml} / \mathrm{d})$ and $\mathrm{Na}_{2} \mathrm{SO}_{4}(18.2 \pm 1.5$ $\mathrm{ml} / \mathrm{d})$ animals compared with control $(11.4 \pm 0.9 \mathrm{ml} / \mathrm{d}, P<0.03$ vs. $\left(\mathrm{NH}_{4}\right)_{2} \mathrm{SO}_{4}$ and $\mathrm{Na}_{2} \mathrm{SO}_{4}$ groups).

Plasma and urine changes induced by drinking solutions. Plasma $\mathrm{HCO}_{3}$ was not different from the respective baseline value in $\left(\mathrm{NH}_{4}\right)_{2} \mathrm{SO}_{4}(25.6 \pm 1.4$ vs. $25.9 \pm 1.4 \mathrm{mM}$, respectively, $P=\mathrm{NS})$, control (25.5 \pm 1.6 vs. $25.7 \pm 1.5 \mathrm{mM}$, respectively, $P=$ NS), or $\mathrm{Na}_{2} \mathrm{SO}_{4}(25.9 \pm 1.3$ vs. $25.0 \pm 1.4 \mathrm{mM}$, respectively, $P=$ NS) animals. Urine $\mathrm{pH}$ decreased compared with its baseline in $\left(\mathrm{NH}_{4}\right)_{2} \mathrm{SO}_{4}(5.32 \pm 0.06$ vs. $6.17 \pm 0.05$, respectively, $P<$ $0.001)$ and $\mathrm{Na}_{2} \mathrm{SO}_{4}(5.45 \pm 0.06$ vs. $6.10 \pm 0.05$, respectively, $P<$ $0.002)$, but not in control $(6.15 \pm 0.05$ vs. $6.16 \pm 0.05$, respectively, $P=\mathrm{NS}$ ) animals. Cumulative 7 -d NAE was higher in $\left(\mathrm{NH}_{4}\right)_{2} \mathrm{SO}_{4}$ compared with control $(42.2 \pm 3.1$ vs. $19.0 \pm 1.8$ $\mathrm{meq} / 7 \mathrm{~d}$, respectively, $P<0.002)$, but was not different from control in $\mathrm{Na}_{2} \mathrm{SO}_{4}(24.2 \pm 2.1 \mathrm{meq} / 7 \mathrm{~d}, P=0.22)$ animals.

RIF ET-1 addition. Fig. 1 shows higher RIF ET-1 addition in $\left(\mathrm{NH}_{4}\right)_{2} \mathrm{SO}_{4}$ animals compared with control $(480 \pm 51$ vs. $293 \pm 32$ fmol g kidney $\mathrm{wt}^{-1} \mathrm{~min}^{-1}$, respectively, $P<0.05$ ), but that for $\mathrm{Na}_{2} \mathrm{SO}_{4}$ animals $\left(275 \pm 23 \mathrm{fmol} \mathrm{g}\right.$ kidney $\left.\mathrm{wt}^{-1} \mathrm{~min}^{-1}\right)$ was not different from control.

Effect of endothelin receptor inhibition on systemic blood pressure. Mean blood pressure of micropunctured animals was similar among bosentan-infused, BQ-123-infused, and baseline $\left(\mathrm{NH}_{4}\right)_{2} \mathrm{SO}_{4}(110 \pm 3,108 \pm 3$, and $115 \pm 4 \mathrm{mmHg}$, respectively), control $(111 \pm 4,112 \pm 3$, and $116 \pm 3 \mathrm{mmHg}$, respectively), and $\mathrm{Na}_{2} \mathrm{SO}_{4}$ animals $(107 \pm 3,113 \pm 3$, and $117 \pm 4 \mathrm{mmHg}$, respectively, $P=\mathrm{NS}$ ).

Micropuncture data. Because the $\left(\mathrm{NH}_{4}\right)_{2} \mathrm{SO}_{4}$-induced increased urine NAE was accompanied by augmented RIF ET-1 addition, we investigated whether endothelin receptor antagonism in vivo influenced the augmented distal tubule acidification induced by dietary $\left(\mathrm{NH}_{4}\right)_{2} \mathrm{SO}_{4}(3)$. Plasma electrolyte and acid-base composition including arterial and stellate vessel plasma $\mathrm{HCO}_{3}$ were not different among groups (data not shown). Table II depicts the effect of bosentan, a nonspecific endothelin receptor antagonist (10), and BQ-123, an $\mathrm{ET}_{\mathrm{A}}$ selective antagonist (11), on distal tubule $\mathrm{HCO}_{3}$ transport in situ. In previous studies, $\left(\mathrm{NH}_{4}\right)_{2} \mathrm{SO}_{4}$ animals had lower late distal fluid $\mathrm{HCO}_{3}$ and lower $\mathrm{HCO}_{3}$ delivery to this nephron segment than control (3). In the present studies, Table II shows that late distal tubule fluid $\mathrm{HCO}_{3}$ was higher than baseline in $\left(\mathrm{NH}_{4}\right)_{2} \mathrm{SO}_{4}$ animals given bosentan but not BQ-123. Late distal tubule fluid $\mathrm{HCO}_{3}$ was higher in bosentan-treated compared with baseline $\left(\mathrm{NH}_{4}\right)_{2} \mathrm{SO}_{4}$ animals despite similar fluid flows ( $2.0 \pm 0.2$ vs. $1.8 \pm 0.2 \mathrm{nl} / \mathrm{min}$, respectively, $P=\mathrm{NS}$ ) and tubular fluid-to-plasma inulin ratios $(14.1 \pm 1.8$ vs. $14.8 \pm 1.9$, respectively, $P=\mathrm{NS}$ ). By contrast, early and late distal tubule $\mathrm{HCO}_{3}$ delivery as well as net $\mathrm{HCO}_{3}$ reabsorption were not different

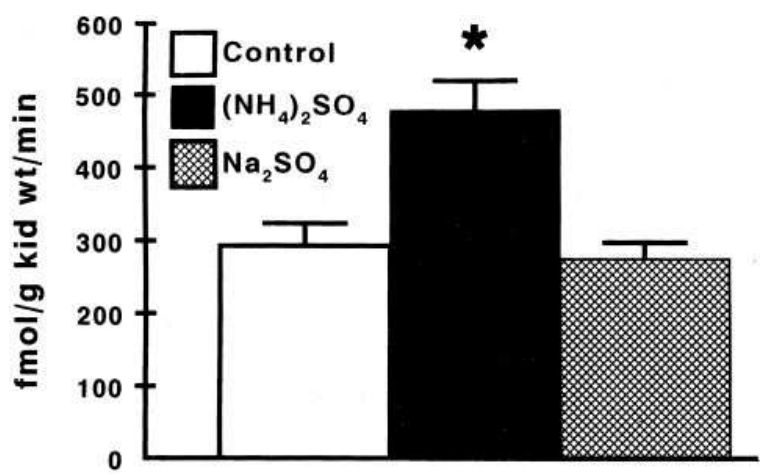

Figure 1. Renal interstitial fluid ET-1 addition in control (open bar), $\left(\mathrm{NH}_{4}\right)_{2} \mathrm{SO}_{4}$ (solid bar), and $\mathrm{Na}_{2} \mathrm{SO}_{4}$ (hatched bar) animals. $* P<0.05$ vs. control. 


\begin{tabular}{|c|c|c|c|c|c|}
\hline & $\mathrm{ED} \mathrm{HCO}_{3}$ & $\mathrm{LD} \mathrm{HCO}_{3}$ & $\mathrm{ED} \mathrm{HCO}_{3}$ delivery & $\mathrm{LD} \mathrm{HCO}_{3}$ delivery & Net $\mathrm{HCO}_{3}$ reabsorption \\
\hline & \multicolumn{2}{|c|}{$m M$} & \multicolumn{3}{|c|}{$\mathrm{pmol} / \mathrm{min}$} \\
\hline \multicolumn{6}{|l|}{$\left(\mathrm{NH}_{4}\right)-\mathrm{SO}_{4}$ animals } \\
\hline$\left(\mathrm{NH}_{4}\right)_{2} \mathrm{SO}_{4}(n=4)$ & $5.4 \pm 0.3$ & $2.0 \pm 0.1$ & $31.9 \pm 2.2$ & $3.6 \pm 0.5$ & $28.2 \pm 2.2$ \\
\hline$\left(\mathrm{NH}_{4}\right)_{2} \mathrm{SO}_{4}+$ bosentan $(n=4)$ & $5.3 \pm 0.3$ & $3.1 \pm 0.2^{*}$ & $31.7 \pm 2.1$ & $6.2 \pm 0.9$ & $25.4 \pm 2.0$ \\
\hline$\left(\mathrm{NH}_{4}\right)_{2} \mathrm{SO}_{4}+\mathrm{BQ}-123(n=4)$ & $5.1 \pm 0.3$ & $1.8 \pm 0.1$ & $31.0 \pm 2.1$ & $3.4 \pm 0.4$ & $27.6 \pm 2.2$ \\
\hline \multicolumn{6}{|l|}{ Control animals } \\
\hline $\mathrm{H}_{2} \mathrm{O}(n=5)$ & $5.6 \pm 0.4$ & $3.9 \pm 0.2$ & $34.2 \pm 2.5$ & $7.8 \pm 1.1$ & $26.5 \pm 2.2$ \\
\hline $\mathrm{H}_{2} \mathrm{O}+\operatorname{bosentan}(n=4)$ & $5.4 \pm 0.3$ & $4.0 \pm 0.2$ & $32.4 \pm 2.4$ & $8.4 \pm 1.2$ & $23.9 \pm 2.1$ \\
\hline \multicolumn{6}{|l|}{$\mathrm{Na}_{2} \mathrm{SO}_{4}$ animals } \\
\hline $\mathrm{Na}_{2} \mathrm{SO}_{4}(n=4)$ & $5.4 \pm 0.4$ & $3.4 \pm 0.2$ & $32.9 \pm 2.3$ & $6.8 \pm 0.9$ & $26.0 \pm 2.1$ \\
\hline $\mathrm{Na}_{2} \mathrm{SO}_{4}+\operatorname{bosentan}(n=4)$ & $5.5 \pm 0.4$ & $3.8 \pm 0.2$ & $33.6 \pm 2.5$ & $7.6 \pm 1.0$ & $26.1 \pm 1.9$ \\
\hline
\end{tabular}

Values are means \pm SEM; ED, early distal tubule; $\mathrm{LD}$, late distal tubule. Chemical designations represent drinking water content. $* P<0.05$ vs. group without drug.

from baseline in bosentan- or BQ-123-infused $\left(\mathrm{NH}_{4}\right)_{2} \mathrm{SO}_{4}$ animals. Likewise, early distal tubule $\mathrm{HCO}_{3}$ was similar among $\left(\mathrm{NH}_{4}\right)_{2} \mathrm{SO}_{4}$ animals. Table II shows that in situ distal tubule $\mathrm{HCO}_{3}$ transport of bosentan-infused animals was not different from baseline in either control or $\mathrm{Na}_{2} \mathrm{SO}_{4}$ groups.

Decreased luminal $\mathrm{HCO}_{3}$ induced by dietary acid might increase distal nephron $\mathrm{NH}_{4}{ }^{+}$secretion (28) and thereby increase nephron acid excretion as discussed (3). The bosentaninduced increase in late distal tubule $\mathrm{HCO}_{3}$ in $\left(\mathrm{NH}_{4}\right)_{2} \mathrm{SO}_{4}$ animals is consistent with an endothelin role in the reduced late distal $\mathrm{HCO}_{3}$ induced by dietary acid (3). The following studies examined if the bosentan-induced increase in late distal $\mathrm{HCO}_{3}$ in situ was mediated by increased $\mathrm{HCO}_{3}$ secretion, decreased $\mathrm{H}^{+}$secretion, or both. Data from distal tubules perfused in paired fashion with $\mathrm{HCO}_{3}$-free solution (solution 1) depicted in Table III were combined with those from paired perfusions with $\mathrm{HCO}_{3}$-containing solutions 3 and 4 (5 and $10 \mathrm{mM} \mathrm{HCO}$, respectively) in Tables $\mathrm{V}$ and $\mathrm{VI}$ to calculate components of net $\mathrm{HCO}_{3}$ reabsorption (see Methods). In previous studies, $\left(\mathrm{NH}_{4}\right)_{2} \mathrm{SO}_{4}$ animals had lower luminal $\mathrm{HCO}_{3}$ accumulation and lower apparent blood-to-lumen $\mathrm{HCO}_{3}$ permeability than control when perfusing distal tubules with zero- $\mathrm{HCO}_{3}$ solutions (3). Table III shows higher luminal $\mathrm{HCO}_{3}$ accumulation and apparent blood-to-lumen $\mathrm{HCO}_{3}$ permeability in distal tubules of bosentan-treated compared with baseline $\left(\mathrm{NH}_{4}\right)_{2} \mathrm{SO}_{4}$ animals, but these parameters were not different from baseline values in BQ-123-treated $\left(\mathrm{NH}_{4}\right)_{2} \mathrm{SO}_{4}$ animals.
To investigate if the increased luminal $\mathrm{HCO}_{3}$ accumulation and apparent blood-to-lumen $\mathrm{HCO}_{3}$ permeability in the bosentan-treated $\left(\mathrm{NH}_{4}\right)_{2} \mathrm{SO}_{4}$ animals was mediated by increased passive blood-to-lumen $\mathrm{HCO}_{3}$ permeability (see Methods), distal tubules were perfused with an $\mathrm{HCO}_{3}$ - and $\mathrm{Cl}^{-}$-free solution containing acetazolamide (solution 2). Table IV shows that when perfusing with this solution, luminal $\mathrm{HCO}_{3}$ accumulation and apparent blood-to-lumen $\mathrm{HCO}_{3}$ permeability were not different between bosentan-treated and baseline $\left(\mathrm{NH}_{4}\right)_{2} \mathrm{SO}_{4}$ animals. These data show that passive blood-to-lumen $\mathrm{HCO}_{3}$ permeability is similar in bosentan-infused and baseline $\left(\mathrm{NH}_{4}\right)_{2} \mathrm{SO}_{4}$ animals.

The next microperfusion studies investigated the effect of endothelin receptor antagonism on net $\mathrm{HCO}_{3}$ reabsorption as well as on calculated $\mathrm{HCO}_{3}$ and $\mathrm{H}^{+}$secretion in distal tubules of $\left(\mathrm{NH}_{4}\right)_{2} \mathrm{SO}_{4}$ animals. Table $\mathrm{V}$ shows that net $\mathrm{HCO}_{3}$ reabsorption in bosentan- and BQ-123-treated $\left(\mathrm{NH}_{4}\right)_{2} \mathrm{SO}_{4}$ animals was not different from that of the baseline $\left(\mathrm{NH}_{4}\right)_{2} \mathrm{SO}_{4}$ group when their distal tubules were perfused with the 5-mM $\mathrm{HCO}_{3}$ solution (solution 3). Fig. 2 shows that calculated $\mathrm{HCO}_{3}$ secretion (see Methods) was higher in bosentan-infused compared with baseline $\left(\mathrm{NH}_{4}\right)_{2} \mathrm{SO}_{4}$ animals $(-4.7 \pm 0.5$ vs. $-2.4 \pm 0.3$ pmol $\left.\mathrm{mm}^{-1} \cdot \mathrm{min}^{-1}, P<0.03\right)$, but that for the BQ-123-infused $\left(\mathrm{NH}_{4}\right)_{2} \mathrm{SO}_{4}$ animals was not $\left(-2.0 \pm 0.2 \mathrm{pmol} \mathrm{mm} \mathrm{mm}^{-1} \cdot \mathrm{min}^{-1}, P=\right.$ NS). By contrast, Fig. 3 shows that calculated $\mathrm{H}^{+}$secretion was not different in the bosentan-infused compared with the baseline $\left(\mathrm{NH}_{4}\right)_{2} \mathrm{SO}_{4}$ animals $\left(22.0 \pm 2.0\right.$ vs. $24.0 \pm 2.1 \mathrm{pmol} \mathrm{mm} \mathrm{mm}^{-1}$.

Table III. Net Blood-to-Lumen $\mathrm{HCO}_{3}$ Fluxes and Permeabilities in Distal Tubules of $\left(\mathrm{NH}_{4}\right)_{2} \mathrm{SO}_{4}$ Animals Perfused with Solution 1 (Zero $\mathrm{HCO}_{3}$ )

\begin{tabular}{|c|c|c|c|c|c|c|c|c|}
\hline \multirow{2}{*}{$\begin{array}{c}\text { Tubule } \\
\text { length } \mathrm{mm}\end{array}$} & \multicolumn{2}{|c|}{ Flow rate $\mathrm{nl} / \mathrm{min}$} & \multicolumn{3}{|c|}{$\mathrm{HCO}_{3} \mathrm{mM}$} & \multirow[b]{2}{*}{$\mathrm{PD} \mathrm{mV}$} & \multirow{2}{*}{$\begin{array}{c}\mathrm{J}_{\mathrm{HCO} 3} \\
\mathrm{pmol} / \mathrm{mm} \cdot \mathrm{min}^{-1}\end{array}$} & \multirow{2}{*}{$\begin{array}{c}\text { Apparent } \\
\text { permeability } \\
\times 10^{-7} \mathrm{~cm}^{2} / \mathrm{s}\end{array}$} \\
\hline & Perfusion & $\mathrm{J}_{\mathrm{v}}$ & Initial & Collected & log mean gradient & & & \\
\hline \multicolumn{9}{|c|}{$\left(\mathrm{NH}_{4}\right)_{2} \mathrm{SO}_{4}(n=4)$} \\
\hline $1.06 \pm 0.03$ & $5.9 \pm 0.2$ & $0.03 \pm 0.01$ & $1.3 \pm 0.1$ & $1.8 \pm 0.2$ & $28.8 \pm 1.2$ & $-13.1 \pm 1.2$ & $-2.7 \pm 0.3$ & $0.20 \pm 0.03$ \\
\hline \multicolumn{9}{|c|}{$\left(\mathrm{NH}_{4}\right)_{2} \mathrm{SO}_{4}+$ bosentan $(n=4)$} \\
\hline $1.08 \pm 0.03$ & $6.0 \pm 0.2$ & $0.04 \pm 0.02$ & $1.2 \pm 0.1$ & $2.2 \pm 0.2$ & $28.3 \pm 1.2$ & $-10.2 \pm 1.2$ & $-5.4 \pm 0.6^{*}$ & $0.39 \pm 0.04 *$ \\
\hline \multicolumn{9}{|c|}{$\left(\mathrm{NH}_{4}\right)_{2} \mathrm{SO}_{4}+\mathrm{BQ}-123(n=4)$} \\
\hline $1.02 \pm 0.03$ & $5.9 \pm 0.2$ & $0.01 \pm 0.03$ & $1.3 \pm 0.1$ & $1.7 \pm 0.2$ & $28.7 \pm 1.2$ & $-12.8 \pm 1.2$ & $-2.3 \pm 0.2$ & $0.17 \pm 0.03$ \\
\hline
\end{tabular}

Values are means \pm SEM; chemical designations refer to content of drinking solution. ${ }^{*} P<0.05$ vs. respective $\left(\mathrm{NH}_{4}\right)_{2} \mathrm{SO}_{4}$ group. 
Table IV. Blood-to-Lumen $\mathrm{HCO}_{3}$ Fluxes and Permeabilities in Distal Tubules of $\left(\mathrm{NH}_{4}\right)_{2} \mathrm{SO}_{4}$ Animals Perfused with Solution 2 (Zero $\mathrm{HCO}_{3}$, Zero $\mathrm{Cl}^{-}$with $0.5 \mathrm{mM}$ Acetazolamide)

\begin{tabular}{|c|c|c|c|c|c|c|c|c|}
\hline \multirow{2}{*}{$\begin{array}{l}\text { Tubule } \\
\text { length } \mathrm{mm}\end{array}$} & \multicolumn{2}{|c|}{ Flow rate $\mathrm{nl} / \mathrm{min}$} & \multicolumn{3}{|c|}{$\mathrm{HCO}_{3} \mathrm{mM}$} & \multirow[b]{2}{*}{ PD mV } & \multirow{2}{*}{$\begin{array}{c}\mathrm{J}_{\mathrm{HCO} 3} \\
\mathrm{pmol} / \mathrm{mm} \cdot \mathrm{min}^{-1}\end{array}$} & \multirow{2}{*}{$\begin{array}{c}\text { Passive } \\
\text { permeability } \\
\times 10^{-7} \mathrm{~cm}^{2} / \mathrm{s}\end{array}$} \\
\hline & Perfusion & $\mathrm{J}_{\mathrm{v}}$ & Initial & Collected & log mean gradient & & & \\
\hline \multicolumn{9}{|c|}{$\left(\mathrm{NH}_{4}\right)_{2} \mathrm{SO}_{4}(n=4)$} \\
\hline $1.02 \pm 0.03$ & $5.9 \pm 0.1$ & $-0.01 \pm 0.04$ & $1.3 \pm 0.1$ & $2.0 \pm 0.2$ & $28.7 \pm 0.8$ & $-13.7 \pm 1.3$ & $-4.1 \pm 0.5$ & $0.31 \pm 0.04$ \\
\hline \multicolumn{9}{|c|}{$\left(\mathrm{NH}_{4}\right)_{2} \mathrm{SO}_{4}+$ bosentan $(n=4)$} \\
\hline $1.04 \pm 0.03$ & $6.1 \pm 0.1$ & $0.04 \pm 0.03$ & $1.3 \pm 0.1$ & $1.9 \pm 0.2$ & $28.4 \pm 0.8$ & $-12.5 \pm 1.2$ & $-3.4 \pm 0.4$ & $0.26 \pm 0.03$ \\
\hline
\end{tabular}

Values are means \pm SEM; chemical designations refer to content of drinking solution.

Table V. Bicarbonate Reabsorption by Distal Tubules of $\left(\mathrm{NH}_{4}\right)_{2} \mathrm{SO}_{4}$ Animals Perfused with Solution 3 (5 $\left.\mathrm{mM} \mathrm{HCO}_{3}\right)$

\begin{tabular}{|c|c|c|c|c|c|c|c|}
\hline \multirow{2}{*}{$\begin{array}{l}\text { Tubule } \\
\text { length } \mathrm{mm}\end{array}$} & \multicolumn{2}{|c|}{ Flow rate $\mathrm{nl} / \mathrm{min}$} & \multicolumn{3}{|c|}{$\mathrm{HCO}_{3} \mathrm{mM}$} & \multirow[b]{2}{*}{ PD mV } & \multirow{2}{*}{$\begin{array}{c}\mathrm{Net} \mathrm{HCO}_{3} \\
\text { reabsorption } \\
\text { pmol/mm } / \mathrm{min}^{-1}\end{array}$} \\
\hline & Perfusion & $\mathrm{J}_{\mathrm{v}}$ & Initial & Collected & log mean gradient & & \\
\hline \multicolumn{8}{|c|}{$\left(\mathrm{NH}_{4}\right)_{2} \mathrm{SO}_{4}(n=4)$} \\
\hline $1.02 \pm 0.03$ & $5.9 \pm 0.1$ & $0.03 \pm 0.02$ & $5.6 \pm 0.3$ & $1.9 \pm 0.2$ & $26.6 \pm 0.9$ & $-14.2 \pm 1.2$ & $21.6 \pm 1.8$ \\
\hline \multicolumn{8}{|c|}{$\left(\mathrm{NH}_{4}\right)_{2} \mathrm{SO}_{4}+$ bosentan $(n=4)$} \\
\hline $1.01 \pm 0.03$ & $6.0 \pm 0.1$ & $0.01 \pm 0.03$ & $5.8 \pm 0.3$ & $3.1 \pm 0.3$ & $25.5 \pm 0.8$ & $-12.2 \pm 1.3$ & $17.3 \pm 1.6$ \\
\hline \multicolumn{8}{|c|}{$\left(\mathrm{NH}_{4}\right)_{2} \mathrm{SO}_{4}+\mathrm{BQ}-123(n=4)$} \\
\hline $1.07 \pm 0.03$ & $6.1 \pm 0.2$ & $0.02 \pm 0.01$ & $5.6 \pm 0.3$ & $1.7 \pm 0.2$ & $26.5 \pm 1.0$ & $-14.8 \pm 1.5$ & $22.3 \pm 1.8$ \\
\hline
\end{tabular}

Values are means \pm SEM; chemical designations refer to content of drinking solution.

$\min ^{-1}$, respectively, $\left.P=\mathrm{NS}\right)$. Calculated $\mathrm{H}^{+}$secretion was also not different from baseline in BQ-123-infused $\left(\mathrm{NH}_{4}\right)_{2} \mathrm{SO}_{4}$ animals $\left(24.3 \pm 2.2 \mathrm{pmol} \mathrm{mm} \mathrm{m}^{-1} \cdot \mathrm{min}^{-1}, P=\mathrm{NS}\right.$ vs. respective baseline value). Thus, increased $\mathrm{HCO}_{3}$ secretion contributes to higher late distal tubule $\mathrm{HCO}_{3}$ of bosentan-infused $\left(\mathrm{NH}_{4}\right)_{2} \mathrm{SO}_{4}$ animals.

The final perfusion studies in $\left(\mathrm{NH}_{4}\right)_{2} \mathrm{SO}_{4}$ animals examined if a minimal attainable $\mathrm{HCO}_{3}$ might limit measured net $\mathrm{HCO}_{3}$ reabsorption in distal tubules perfused with $\mathrm{HCO}_{3}$-containing solutions, limiting calculated $\mathrm{H}^{+}$secretion in these animals, and possibly concealing an effect of bosentan on $\mathrm{H}^{+}$secretion. Distal tubules were perfused with solution 4 , containing a higher initial $\mathrm{HCO}_{3}(10 \mathrm{mM})$ than solution 3, as discussed earlier. The data are depicted in Table VI. In previous studies, net

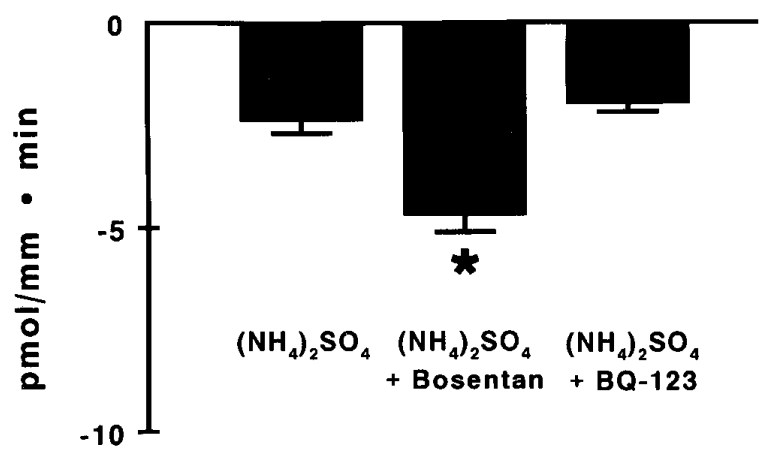

Figure 2. Bicarbonate secretion by distal tubules perfused with solution $3\left(5 \mathrm{mM} \mathrm{HCO}_{3}\right)$ in $\left(\mathrm{NH}_{4}\right)_{2} \mathrm{SO}_{4}$-ingesting animals at baseline and after bosentan $(10 \mathrm{mg} / \mathrm{kg})$ or BQ-123 $(1 \mathrm{mg} / \mathrm{kg}$ bolus followed by 0.1 $\mathrm{mg} \mathrm{kg}{ }^{-1} \mathrm{~min}^{-1}$ ) infusion. $* P<0.05$ vs. $\left(\mathrm{NH}_{4}\right)_{2} \mathrm{SO}_{4}$ (baseline) group.
$\mathrm{HCO}_{3}$ reabsorption was higher in $\left(\mathrm{NH}_{4}\right)_{2} \mathrm{SO}_{4}$ compared with control animals when distal tubules of each were perfused with $10 \mathrm{mM} \mathrm{HCO}_{3}$ solution (3). Table VI shows lower net $\mathrm{HCO}_{3}$ reabsorption in distal tubules of bosentan-infused but not BQ123-infused $\left(\mathrm{NH}_{4}\right)_{2} \mathrm{SO}_{4}$ animals. $\mathrm{H}^{+}$and $\mathrm{HCO}_{3}$ secretion for the $10-\mathrm{mM} \mathrm{HCO}_{3}$ perfusions were calculated as for the $5-\mathrm{mM}$ $\mathrm{HCO}_{3}$ perfusions, using apparent permeability derived from the perfusion with solution 1 (see Methods). Fig. 4 shows that calculated $\mathrm{HCO}_{3}$ secretion was higher in distal tubules of the bosentan-infused compared with the baseline $\left(\mathrm{NH}_{4}\right)_{2} \mathrm{SO}_{4}$ animals perfused with $10 \mathrm{mM} \mathrm{HCO}_{3}(-3.9 \pm 0.4$ vs. $-2.0 \pm 0.2$ pmol $\left.\mathrm{mm}^{-1} \cdot \mathrm{min}^{-1}, P<0.05\right)$, but that for the BQ-123-infused $\left(\mathrm{NH}_{4}\right)_{2} \mathrm{SO}_{4}$ animals was not $\left(-1.7 \pm 0.2 \mathrm{pmol} \mathrm{mm} \mathrm{mm}^{-1} \cdot \mathrm{min}^{-1}, P=\right.$ NS). In contrast with the findings described when perfusing with the $5-\mathrm{mM} \mathrm{HCO}_{3}$ solutions, Fig. 5 shows that bosentaninfused $\left(\mathrm{NH}_{4}\right)_{2} \mathrm{SO}_{4}$ animals had lower calculated $\mathrm{H}^{+}$secretion compared with the baseline value $(27.7 \pm 2.5$ vs. $43.9 \pm 4.0 \mathrm{pmol}$ $\left.\mathrm{mm}^{-1} \cdot \min ^{-1}, P<0.05\right) . \mathrm{H}^{+}$secretion in distal tubules of BQ123-infused $\left(\mathrm{NH}_{4}\right)_{2} \mathrm{SO}_{4}$ animals was not different from the baseline value $\left(42.9 \pm 4.2 \mathrm{pmol} \mathrm{mm} \mathrm{mm}^{-1} \cdot \mathrm{min}^{-1}, P=\mathrm{NS}\right)$. Thus,

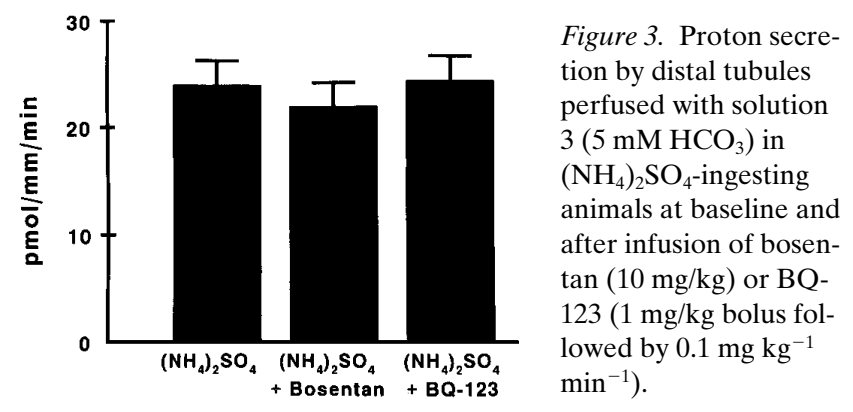


Table VI. Bircarbonate Reabsorption by Distal Tubules $\left(\mathrm{NH}_{4}\right)_{2} \mathrm{SO}_{4}$ Animals Perfused with Solution $4\left(10 \mathrm{mM} \mathrm{HCO}_{3}\right)$

\begin{tabular}{|c|c|c|c|c|c|c|c|}
\hline \multirow{2}{*}{$\begin{array}{l}\text { Tubule } \\
\text { length } \mathrm{mm}\end{array}$} & \multicolumn{2}{|c|}{ Flow rate $\mathrm{nl} / \mathrm{min}$} & \multicolumn{3}{|c|}{$\mathrm{HCO}_{3} \mathrm{mM}$} & \multirow[b]{2}{*}{ PD mV } & \multirow{2}{*}{$\begin{array}{c}\mathrm{Net}^{\mathrm{HCO}_{3}} \\
\text { reabsorption } \\
\text { pmol/mm } / \mathrm{min}^{-1}\end{array}$} \\
\hline & Perfusion & $J_{v}$ & Initial & Collected & log mean gradient & & \\
\hline \multicolumn{8}{|c|}{$\left(\mathrm{NH}_{4}\right)_{2} \mathrm{SO}_{4}(n=3)$} \\
\hline $0.96 \pm 0.03$ & $5.9 \pm 0.2$ & $0.01 \pm 0.03$ & $11.3 \pm 0.2$ & $4.5 \pm 0.2 *$ & $22.3 \pm 1.0$ & $-15.0 \pm 1.4$ & $41.9 \pm 3.8$ \\
\hline \multicolumn{8}{|c|}{$\left(\mathrm{NH}_{4}\right)_{2} \mathrm{SO}_{4}+$ bosentan $(n=4)$} \\
\hline $1.03 \pm 0.04$ & $6.2 \pm 0.3$ & $0.05 \pm 0.04$ & $11.2 \pm 0.3$ & $7.3 \pm 0.3^{*}$ & $21.7 \pm 1.1$ & $-12.9 \pm 1.3$ & $23.8 \pm 2.5^{*}$ \\
\hline \multicolumn{8}{|c|}{$\left(\mathrm{NH}_{4}\right)_{2} \mathrm{SO}_{4}+\mathrm{BQ}-123(n=3)$} \\
\hline $0.98 \pm 0.03$ & $6.0 \pm 0.2$ & $0.04 \pm 0.02$ & $11.1 \pm 0.2$ & $4.4 \pm 0.2$ & $22.3 \pm 1.1$ & $-15.1 \pm 1.5$ & $41.2 \pm 4.2$ \\
\hline
\end{tabular}

Values are means \pm SEM; chemical designations refer to content of drinking solution. $* P<0.05$ vs. respective $\left(\mathrm{NH}_{4}\right)_{2} \mathrm{SO}_{4}$ group.

Table VII. Net Blood-to-Lumen $\mathrm{HCO}_{3}$ Fluxes and Permeabilities in Distal Tubules of Control Animals Perfused with Solution 1 (Zero $\left.\mathrm{HCO}_{3}\right)$

\begin{tabular}{|c|c|c|c|c|c|c|c|c|}
\hline \multirow{2}{*}{$\begin{array}{c}\text { Tubule } \\
\text { length } \mathrm{mm}\end{array}$} & \multicolumn{2}{|c|}{ Flow rate $\mathrm{nl} / \mathrm{min}$} & \multicolumn{3}{|c|}{$\mathrm{HCO}_{3} \mathrm{mM}$} & \multirow[b]{2}{*}{ PD mV } & \multirow{2}{*}{$\begin{array}{c}\mathrm{J}_{\mathrm{HCO} 3} \\
\mathrm{pmol} / \mathrm{mm} \cdot \mathrm{min}^{-1}\end{array}$} & \multirow{2}{*}{$\begin{array}{c}\text { Apparent } \\
\text { permeability } \\
\times 10^{-7} \mathrm{~cm}^{2} / \mathrm{s}\end{array}$} \\
\hline & Perfusion & $\mathrm{J}_{\mathrm{v}}$ & Initial & Collected & log mean gradient & & & \\
\hline \multicolumn{9}{|l|}{$\mathrm{H}_{2} \mathrm{O}(n=5)$} \\
\hline $1.03 \pm 0.03$ & $5.9 \pm 0.2$ & $0.01 \pm 0.03$ & $1.3 \pm 0.1$ & $2.3 \pm 0.2$ & $31.7 \pm 1.2$ & $-12.9 \pm 1.3$ & $-5.7 \pm 0.8$ & $0.39 \pm 0.05$ \\
\hline \multicolumn{9}{|c|}{$\mathrm{H}_{2} \mathrm{O}+\operatorname{bosentan}(n=4)$} \\
\hline $1.05 \pm 0.04$ & $5.9 \pm 0.1$ & $0.02 \pm 0.02$ & $1.2 \pm 0.1$ & $2.4 \pm 0.2$ & $31.7 \pm 1.3$ & $-12.4 \pm 1.2$ & $-6.6 \pm 0.7$ & $0.44 \pm 0.05$ \\
\hline
\end{tabular}

Values are means \pm SEM; chemical designations refer to content of drinking solution.

decreased $\mathrm{H}^{+}$secretory capacity contributes to higher $\mathrm{HCO}_{3}$ at the late distal tubule in situ of bosentan-infused $\left(\mathrm{NH}_{4}\right)_{2} \mathrm{SO}_{4}$ animals.

We next examined the effect of bosentan on $\mathrm{HCO}_{3}$ transport in control animals. Table VII shows no difference in luminal $\mathrm{HCO}_{3}$ accumulation or apparent permeability between bosentan-infused and baseline controls perfused with solution 1 (zero $\mathrm{HCO}_{3}$ ). Because differences in net $\mathrm{HCO}_{3}$ reabsorption between $\left(\mathrm{NH}_{4}\right)_{2} \mathrm{SO}_{4}$ animals and control were evident perfusing with the 10 but not $5 \mathrm{mM} \mathrm{HCO}_{3}$ solution, the $10-\mathrm{mM}$ solution (solution 4) was perfused in distal tubules of control animals to determine if bosentan influenced net $\mathrm{HCO}_{3}$ reabsorption and/or its components $\left(\mathrm{HCO}_{3} / \mathrm{H}^{+}\right.$secretion) in con-

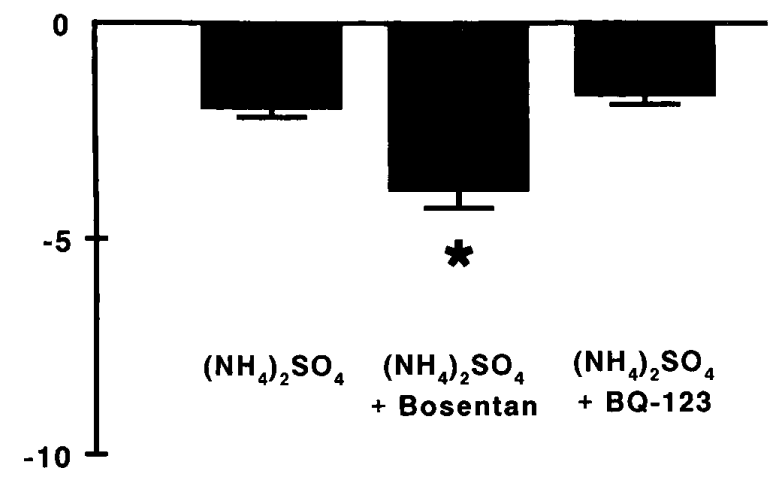

Figure 4. Bicarbonate secretion by distal tubules perfused with solution $4\left(10 \mathrm{mM} \mathrm{HCO}_{3}\right)$ in $\left(\mathrm{NH}_{4}\right)_{2} \mathrm{SO}_{4}$-ingesting animals at baseline and after infusion of bosentan $(10 \mathrm{mg} / \mathrm{kg})$ or BQ-123 $(1 \mathrm{mg} / \mathrm{kg}$ bolus followed by $\left.0.1 \mathrm{mg} \mathrm{kg}^{-1} \mathrm{~min}^{-1}\right)$. $* P<0.05$ vs. $\left(\mathrm{NH}_{4}\right)_{2} \mathrm{SO}_{4}$ (baseline) group. trol animals. Table VIII shows that net $\mathrm{HCO}_{3}$ reabsorption was not different between bosentan-infused and baseline control animals perfused with $10 \mathrm{mM} \mathrm{HCO}_{3}$. Calculated secretion of $\mathrm{HCO}_{3}\left(-5.0 \pm 0.6\right.$ vs. $\left.-4.4 \pm 0.5 \mathrm{pmol} \mathrm{mm} \mathrm{mm}^{-1} \cdot \mathrm{min}^{-1}, P=\mathrm{NS}\right)$ and $\mathrm{H}^{+}\left(20.2 \pm 2.0\right.$ vs. $\left.23.2 \pm 2.2 \mathrm{pmol} \mathrm{mm}^{-1} \cdot \mathrm{min}^{-1}, P=\mathrm{NS}\right)$ were also not different in bosentan-infused and baseline controls, respectively. Thus, endothelin receptor antagonism did not affect distal tubule acidification in control animals.

The final series of microperfusion studies determined if bosentan influenced distal tubule $\mathrm{HCO}_{3}$ transport in $\mathrm{Na}_{2} \mathrm{SO}_{4}$ animals, a group whose urine flow was higher than control but comparable to that for $\left(\mathrm{NH}_{4}\right)_{2} \mathrm{SO}_{4}$ animals. This higher urine flow might itself increase urine ET-1 excretion (9) and thereby influence distal tubule acidification independent of dietary acid intake. Table IX shows no difference in luminal $\mathrm{HCO}_{3}$ accumulation or apparent permeability between bosentan-infused and baseline $\mathrm{Na}_{2} \mathrm{SO}_{4}$ animals perfused with solution 1 (zero $\mathrm{HCO}_{3}$ ). As with the control animals, the $10-\mathrm{mM} \mathrm{HCO}_{3}$ solution (solution 4) was used in distal tubules of $\mathrm{Na}_{2} \mathrm{SO}_{4}$ animals

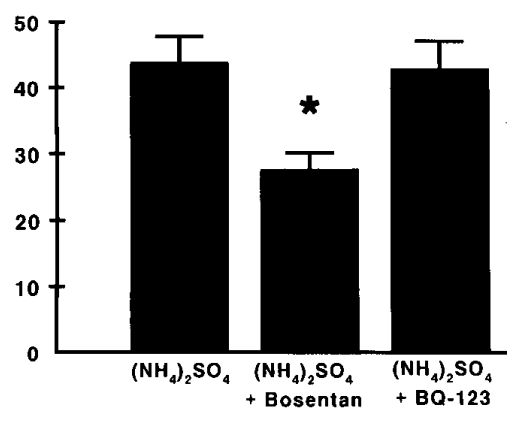

Figure 5. Proton secretion by distal tubules perfused with solution 4 $\left(10 \mathrm{mM} \mathrm{HCO}_{3}\right)$ in $\left(\mathrm{NH}_{4}\right)_{2} \mathrm{SO}_{4}$-ingesting animals at baseline and after infusion of bosentan $(10 \mathrm{mg} / \mathrm{kg})$ or BQ-123 ( $1 \mathrm{mg} / \mathrm{kg}$ bolus followed by $0.1 \mathrm{mg} \mathrm{kg}^{-1} \mathrm{~min}^{-1}$ ). $* P<0.05$ vs. $\left(\mathrm{NH}_{4}\right)_{2} \mathrm{SO}_{4}$ (baseline) group. 
Table VIII. Bicarbonate Reabsorption by Distal Tubules of Control Animals Perfused with Solution 4 (10 mM HCO $\mathrm{m}_{3}$

\begin{tabular}{|c|c|c|c|c|c|c|c|}
\hline \multirow{2}{*}{$\begin{array}{c}\text { Tubule } \\
\text { length } \mathrm{mm}\end{array}$} & \multicolumn{2}{|c|}{ Flow rate $\mathrm{nl} / \mathrm{min}$} & \multicolumn{3}{|c|}{$\mathrm{HCO}_{3} \mathrm{mM}$} & \multirow[b]{2}{*}{ PD mV } & \multirow{2}{*}{$\begin{array}{c}\mathrm{Net}_{\mathrm{HCO}_{3}} \\
\text { reabsorption } \\
\text { pmol/mm·min }\end{array}$} \\
\hline & Perfusion & $J_{v}$ & Initial & Collected & log mean gradient & & \\
\hline \multicolumn{8}{|l|}{$\mathrm{H}_{2} \mathrm{O}(n=4)$} \\
\hline $1.04 \pm 0.03$ & $5.9 \pm 0.1$ & $0.01 \pm 0.03$ & $10.6 \pm 0.5$ & $7.3 \pm 0.3$ & $24.5 \pm 1.3$ & $-13.2 \pm 1.5$ & $18.8 \pm 1.9$ \\
\hline \multicolumn{8}{|c|}{$\mathrm{H}_{2} \mathrm{O}+\operatorname{bosentan}(n=4)$} \\
\hline $1.02 \pm 0.03$ & $5.9 \pm 0.1$ & $0.01 \pm 0.03$ & $10.3 \pm 0.4$ & $7.7 \pm 0.3$ & $24.5 \pm 1.3$ & $-12.8 \pm 1.2$ & $15.1 \pm 1.5$ \\
\hline
\end{tabular}

Values are means \pm SEM; chemical designations refer to content of drinking solution.

Table IX. Net Blood-to-Lumen $\mathrm{HCO}_{3}$ Fluxes and Permeabilities in Distal Tubules of $\mathrm{Na}_{2} \mathrm{SO}_{4}$ Animals Perfused with Solution 1 (Zero $\left.\mathrm{HCO}_{3}\right)$

\begin{tabular}{|c|c|c|c|c|c|c|c|c|}
\hline \multirow{2}{*}{$\begin{array}{c}\text { Tubule } \\
\text { length } \mathrm{mm}\end{array}$} & \multicolumn{2}{|c|}{ Flow rate $\mathrm{nl} / \mathrm{min}$} & \multicolumn{3}{|c|}{$\mathrm{HCO}_{3} \mathrm{mM}$} & \multirow[b]{2}{*}{ PD mV } & \multirow{2}{*}{$\begin{array}{c}\mathrm{J}_{\mathrm{HCO} 3} \\
\mathrm{pmol} / \mathrm{mm} \cdot \mathrm{min}^{-1}\end{array}$} & \multirow{2}{*}{$\begin{array}{c}\text { Apparent } \\
\text { permeability } \\
\times 10^{-7} \mathrm{~cm}^{2} / \mathrm{s}\end{array}$} \\
\hline & Perfusion & $\mathrm{J}_{\mathrm{v}}$ & Initial & Collected & log mean gradient & & & \\
\hline \multicolumn{9}{|c|}{$\mathrm{Na}_{2} \mathrm{SO}_{4}(n=4)$} \\
\hline $0.93 \pm 0.04$ & $5.9 \pm 0.2$ & $-0.03 \pm 0.01$ & $1.3 \pm 0.1$ & $2.0 \pm 0.2$ & $30.3 \pm 1.1$ & $-16.2 \pm 1.5$ & $-4.5 \pm 0.6$ & $0.34 \pm 0.05$ \\
\hline \multicolumn{9}{|c|}{$\mathrm{Na}_{2} \mathrm{SO}_{4}+\operatorname{bosentan}(n=4)$} \\
\hline $0.97 \pm 0.04$ & $6.1 \pm 0.2$ & $0.04 \pm 0.02$ & $1.4 \pm 0.1$ & $2.2 \pm 0.2$ & $30.0 \pm 1.1$ & $-15.1 \pm 1.3$ & $-5.2 \pm 0.7$ & $0.38 \pm 0.05$ \\
\hline
\end{tabular}

Values are means \pm SEM; chemical designations refer to content of drinking solution.

to determine if bosentan influenced net $\mathrm{HCO}_{3}$ reabsorption and/or its components in this group. Table $\mathrm{X}$ shows that net $\mathrm{HCO}_{3}$ reabsorption was not different between bosentaninfused and baseline $\mathrm{Na}_{2} \mathrm{SO}_{4}$ animals. Calculated secretion of $\mathrm{HCO}_{3}\left(-3.8 \pm 0.5\right.$ vs. $\left.-3.4 \pm 0.4 \mathrm{pmol} \mathrm{mm}^{-1} \cdot \mathrm{min}^{-1}, P=\mathrm{NS}\right)$ and $\mathrm{H}^{+}\left(23.8 \pm 2.2\right.$ vs. $\left.29.4 \pm 2.6 \mathrm{pmol} \mathrm{mm} \mathrm{mm}^{-1} \cdot \mathrm{min}^{-1}, P=\mathrm{NS}\right)$ were also not different in bosentan-infused and baseline $\mathrm{Na}_{2} \mathrm{SO}_{4}$ animals, respectively. Thus, endothelin receptor antagonism did not affect distal tubule acidification in $\mathrm{Na}_{2} \mathrm{SO}_{4}$ animals.

Effect of endothelin receptor inhibition on NAE. Because bosentan decreased acidification in the distal tubule of $\left(\mathrm{NH}_{4}\right)_{2} \mathrm{SO}_{4}$ animals, we investigated if this receptor antagonist reduced NAE. 12-h NAE after administration of the same bosentan dose $(10 \mathrm{mg} / \mathrm{kg}$ i.v.) that had been given to micropunctured animals was not different in bosentan-infused compared with vehicle-infused $\left(\mathrm{NH}_{4}\right)_{2} \mathrm{SO}_{4}(3.2 \pm 0.5$ vs. $4.1 \pm 0.6$ meq/12 h, respectively, $P=\mathrm{NS})$, control $(1.7 \pm 0.3$ vs. $1.7 \pm 0.2$ meq/12 h, respectively, $P=\mathrm{NS})$, or $\mathrm{Na}_{2} \mathrm{SO}_{4}(1.7 \pm 0.2$ vs. $1.8 \pm 0.2 \mathrm{meq} / 12 \mathrm{~h}$, respectively, $P=\mathrm{NS}$ ) animals.

\section{Discussion}

Many investigations employing a variety of techniques have defined effector mechanisms by which the kidney increases acidification in response to dietary acid (26). Such investigations show an important role of increased distal nephron acidification in this response (1-3, 27-29). The distal tubule of animals ingesting dietary acid have augmented $\mathrm{H}^{+}$secreting capacity (1-3) and reduced $\mathrm{HCO}_{3}$ delivery to the terminal distal nephron $(3,30)$, the latter facilitating increased titration of non- $\mathrm{HCO}_{3}$ buffers $(28,31)$. Recent studies from our laboratory show that reduced distal tubule $\mathrm{HCO}_{3}$ secretion contributes to the decreased terminal distal nephron $\mathrm{HCO}_{3}$ delivery induced by dietary acid (3). Thus, dietary acid alters both components of distal tubule net $\mathrm{HCO}_{3}$ reabsorption $\left(\mathrm{H}^{+} / \mathrm{HCO}_{3}\right.$ secretion) in a direction that increases acidification, but the immediate stimulus that induces this response is not known. Much less is understood about the mechanisms that induce distal nephron epithelia to respond in this way to a dietary acid challenge. Dietary acid-induced alterations in body fluid acid-

Table X. Bicarbonate Reabsorption by Distal Tubules of $\mathrm{Na}_{2} \mathrm{SO}_{4}$ Animals Perfused with Solution 4 (10 $\left.\mathrm{mM} \mathrm{HCO}_{3}\right)$

\begin{tabular}{|c|c|c|c|c|c|c|c|}
\hline \multirow{2}{*}{$\begin{array}{c}\text { Tubule } \\
\text { length } \mathrm{mm}\end{array}$} & \multicolumn{2}{|c|}{ Flow rate $\mathrm{nl} / \mathrm{min}$} & \multicolumn{3}{|c|}{$\mathrm{HCO}_{3} \mathrm{mM}$} & \multirow[b]{2}{*}{ PD mV } & \multirow{2}{*}{$\begin{array}{c}\mathrm{Net} \mathrm{HCO}_{3} \\
\text { reabsorption } \\
\mathrm{pmol} / \mathrm{mm} \cdot \mathrm{min}^{-1}\end{array}$} \\
\hline & Perfusion & $\mathrm{J}_{\mathrm{v}}$ & Initial & Collected & $\log$ mean gradient & & \\
\hline \multicolumn{8}{|c|}{$\mathrm{Na}_{2} \mathrm{SO}_{4}(n=4)$} \\
\hline $1.01 \pm 0.05$ & $6.1 \pm 0.1$ & $0.05 \pm 0.03$ & $10.9 \pm 0.5$ & $6.7 \pm 0.4$ & $23.1 \pm 1.3$ & $-15.9 \pm 1.5$ & $25.6 \pm 2.3$ \\
\hline \multicolumn{8}{|c|}{$\mathrm{Na}_{2} \mathrm{SO}_{4}+$ bosentan $(n=4)$} \\
\hline $0.98 \pm 0.03$ & $5.8 \pm 0.2$ & $-0.04 \pm 0.02$ & $10.7 \pm 0.6$ & $7.2 \pm 0.4$ & $22.8 \pm 1.3$ & $-15.8 \pm 1.6$ & $20.4 \pm 1.8$ \\
\hline
\end{tabular}

Values are means \pm SEM; chemical designations refer to content of drinking solution. 
base parameters might increase distal nephron acidification directly. Although changes in environmental acid-base parameters can induce predictable alterations in acidification of renal epithelia studied in vitro $(32,33)$, it is clear that sustained changes in plasma $(3,24)$ or intracellular $(4)$ acid-base parameters are not necessary to maintain altered renal epithelial acidification $(3,4,24)$. Alternative or additional mechanisms include diet-induced modification of the level and/or activity of secretory substances that modify renal epithelial acidification. The present studies tested the hypothesis that endothelin mediates increased distal tubule acidification induced by dietary acid. This hypothesis derives from studies showing that ET-1 increases NHE-3 activity in cultured renal epithelial cells (8) and decreases distal tubule $\mathrm{HCO}_{3}$ secretion induced by dietary $\mathrm{HCO}_{3}$ (7). The data show that animals given dietary acid have greater ET-1 addition to RIF. Furthermore, B- but not A-type endothelin receptor inhibition blunts decreased $\mathrm{HCO}_{3}$ secretion and increased $\mathrm{H}^{+}$-secreting capacity induced by dietary acid. Endothelin receptor inhibition had no measurable effect on distal tubule acidification in control animals, suggesting that endothelin contributes less to the "tonic" level of distal tubule acidification under control conditions. The data support the hypothesis that endothelin mediates increased distal tubule acidification induced by dietary acid.

Although the endothelins were initially noted for their vasoactive effects (34), these agents also modulate epithelial transport by inhibiting the amiloride-sensitive $\mathrm{Na}^{+}$channel (35) and ADH-mediated $\mathrm{H}_{2} \mathrm{O}$ reabsorption (36) in collecting tubules. ET-1 also stimulates the $\mathrm{Na}^{+} / \mathrm{H}^{+}$exchanger in renal cortical membrane vesicles (37) and the NHE-3 isoform in renal epithelial cells (8), supporting a possible endothelin role in modulating renal acidification in vivo. The NHE performs much of the $\mathrm{H}^{+}$secretion in the rat distal tubule accessible to micropuncture (38). ET-1 also inhibits agonist-stimulated increases in cellular cyclic AMP levels in renal epithelium (36), a cellular second messenger whose increase is associated with augmented $\mathrm{HCO}_{3}$ secretion in cortical collecting tubules (39) and with inhibited NHE activity in renal brush border membranes (40). Preliminary studies show that ET-1 decreases distal tubule $\mathrm{HCO}_{3}$ secretion induced by dietary $\mathrm{HCO}_{3}(7)$, an important response to this dietary maneuver (24). By contrast, ET-1 inhibited net $\mathrm{HCO}_{3}$ reabsorption in proximal straight tubules (41), suggesting distinct ET-1 effects on acidification in this nephron segment. The present studies show that endothelin receptor inhibition blunts decreased $\mathrm{HCO}_{3}$ secretion and increased $\mathrm{H}^{+}$secretion in the distal tubule induced by dietary acid (3). The data suggest that endogenous endothelins mediate the decreased $\mathrm{HCO}_{3}$ secretion and increased $\mathrm{H}^{+}$secretion induced by dietary acid.

The present studies show that dietary acid increases ET-1 addition to RIF, a fluid compartment in direct communication with basolateral surfaces of cortical renal epithelium. This anatomical arrangement would permit endothelins secreted into RIF to modulate cortical epithelial transport through endothelin receptors on the surface of cultured canine cells of distal nephron origin (42). Furthermore, rat cortical collecting tubules contain mRNA for the B-type endothelin receptor (43), which the present studies suggest mediates the described actions of endogenous endothelins. Endothelins in the RIF might derive from collecting tubule epithelium (5) or endothelium of the renal microvasculature $(6,44)$. The latter is separated from distal tubules in vivo by only a very narrow interstitial space
(45), permitting paracrine or autocrine communication among cell types. In addition, microdissected cortical collecting tubules contain ET-1 mRNA $(43,46)$, and ET-1 is on the endothelial surfaces of peritubular capillaries (44). The present studies show that dietary acid increases RIF ET-1 addition and suggest that the added ET-1 subsequently increases distal tubule acidification.

Calculated $\mathrm{HCO}_{3}$ and $\mathrm{H}^{+}$secretion were not different in bosentan-infused control animals, in contrast with the $\left(\mathrm{NH}_{4}\right)_{2} \mathrm{SO}_{4}$ animals in which bosentan increased $\mathrm{HCO}_{3}$ secretion and decreased $\mathrm{H}^{+}$secretory capacity. Although a higher bosentan dose might influence control $\mathrm{HCO}_{3} / \mathrm{H}^{+}$secretion, the data suggest that endogenous endothelins have a greater influence on basal distal tubule acidification in acid-ingesting compared with control animals. By contrast, endogenous endothelins apparently contribute less than intrinsic, neural, or other autocrine/paracrine mechanisms to basal distal tubule acidification. Nevertheless, the same bosentan dose that reduced distal nephron acidification in $\left(\mathrm{NH}_{4}\right)_{2} \mathrm{SO}_{4}$ animals did not reduce augmented NAE in these animals. This suggests less endothelin effect on acidification and/or less sensitivity to the administered bosentan dose in more terminal nephron segments.

The present studies support that endogenous endothelins inhibit distal tubule $\mathrm{HCO}_{3}$ secretion and stimulate $\mathrm{H}^{+}$secretion in this nephron segment, but do not indicate the cellular signaling mechanisms that mediate these effects. Increased cellular cAMP levels are associated with stimulated $\mathrm{HCO}_{3}$ secretion by the collecting tubule (39) and with inhibited $\mathrm{Na}^{+} / \mathrm{H}^{+}$ exchange in brush border membranes (40). In addition, ET-1 inhibits agonist-induced increases in cortical collecting tubule cAMP (36), complimenting the ET-1 effects on distal tubule $\mathrm{HCO}_{3}$ secretion. Furthermore, prostacyclin $\left(\mathrm{PGI}_{2}\right)$, an agent that increases cellular cAMP levels in distal nephron epithelia (47), increases distal tubule $\mathrm{HCO}_{3}$ secretion (48). Yet, ET-1 also stimulates $\mathrm{Ca}^{2+}$ release from internal stores and its entry into cortical collecting duct cells (49), consistent with activation of phospholipase $C$ (50). In addition, activation of the $\mathrm{B}$-type endothelin receptor leads to generation of nitric oxide through a tyrosine kinase-dependent and $\mathrm{Ca}^{2+} /$ calmodulindependent pathway (51). Thus, ET-1 might alter $\mathrm{HCO}_{3} / \mathrm{H}^{+}$secretion by these and possibly other mechanisms.

In summary, the present studies show that reduced $\mathrm{HCO}_{3}$ secretion and increased $\mathrm{H}^{+}$secretory capacity in the distal tubule induced by dietary acid is associated with increased ET-1 addition to RIF. Furthermore, inhibition of B- but not A-type endothelin receptors blunts the reduced $\mathrm{HCO}_{3}$ secretion and increased $\mathrm{H}^{+}$secretory capacity induced by dietary acid, but does not affect distal tubule acidification in control animals. The data suggest that endogenous endothelins mediate increased distal tubule acidification induced by dietary acid but contribute less to basal acidification in this segment.

\section{Acknowledgments}

We are grateful to Mrs. Geraldine Tasby and Ms. Cathey Hudson for expert technical assistance, and to Neil A. Kurtzman for his continued support. We are also grateful to Martine Clozel, M.D. for generously providing bosentan, without which these studies would not have been possible.

This work was supported by funds from National Institutes of Health grant 5-RO1-DK 36199-10 (N.A. Kurtzman, principle investigator) and from the Texas Tech University Health Sciences Center. 


\section{References}

1. Levine, D.Z. 1985. An in vivo microperfusion study of distal tubule bicarbonate reabsorption in normal and ammonium chloride rats. J. Clin. Invest. 75 : 588-595

2. Kunau, R.T., and K.A. Walker. 1987. Total $\mathrm{CO}_{2}$ absorption in the distal tubule of the rat. Am. J. Physiol. 252:F468-F473.

3. Wesson, D.E. 1996. Reduced bicarbonate secretion mediates increased distal tubule acidification induced by dietary acid. Am. J. Physiol. 271:F670F678.

4. Horie, S., O. Moe, A. Tejedor, and R.J. Alpern. 1990. Preincubation in acid medium increases $\mathrm{Na} / \mathrm{H}$ antiporter activity in cultured renal proximal tubule cells. Proc. Natl. Acad. Sci. USA. 87:4742-4745.

5. Kohan, D.E. 1991. Endothelin synthesis by rabbit renal tubule cells. Am. J. Physiol. 261:F221-F226.

6. Wesson, D.E., J. Simoni, and D.F. Green. 1996. Acidic extracellular pH increases Endothelin-1 secretion by human endothelial cells of glomerular but not aortic origin. J. Invest. Med. 44:336a. (Abstr.)

7. Wesson, D.E., and G.M. Dolson. 1996. Endothelin inhibits distal tubule $\mathrm{HCO}_{3}$ secretion induced by dietary $\mathrm{HCO}_{3}$. American Physiological Society Conference, Snowmass, CO. July 12-14. (Abstr.)

8. Chu, T.-S., Y. Peng, A. Cano, M. Yanagisawa, and R.J. Alpern. 1996. Endothelin $_{B}$ receptor activates NHE-3 by a $\mathrm{Ca}^{2+}$-dependent pathway in OKP cells. J. Clin. Invest. 97:1454-1462.

9. Zeiler, M., B.M. Loffler, H.A. Block, and G. Thiel. 1995. ET-1 excretion is flow-dependent in kidney donors and transplant recipients. J. Cardiovasc. Pharmacol. 26(Suppl. 3):S513-S515.

10. Clozel, M., V. Breu, G. Gray, B. Kalina, B.-M. Loffler, K. Burri, J.-M. Cassal, G. Hirth, M. Muller, W. Neidhart, and H. Ramuz. 1994. Pharmacological characterization of Bosentan, a new potent orally active nonpeptide endothelin receptor antagonist. J. Pharmacol. Exp. Ther. 270:228-235.

11. Ihara, M., K. Noguchi, T. Fukuroda, S. Tsuchida, S Kimura, T. Fukami, K. Ishikawa, M. Nishikibe, and M. Yano. 1992. Biological profiles of highly potent novel endothelin antagonists selective for the ETA receptor. Life Sci. 50: 247-255.

12. Kivlighn, S.D., R.A. Gabel, and P.K.S. Siegl. 1994. Effects of BQ-123 on renal function and acute cyclosporin-induced renal dysfunction. Kidney Int. 45: $131-136$

13. Wesson, D.E. 1990. Dietary bicarbonate reduces rat distal nephron acidification evaluated in situ. Am. J. Physiol. 258:F870-F876.

14. Siragy, H.M., M.M. Ibrahim, A.A. Jaffa, R. Mayfield, and H.S. Margolius. 1994. Rat renal interstitial bradykinin, prostaglandin $\mathrm{E}_{2}$, and cyclic guanosine 3', 5'-monophosphate. Effects of altered sodium intake. Hypertension (Dallas). 23:1068-1070.

15. Benigni, A., N. Perico, F. Gaspari, C. Zoja, L. Bellizzi, M. Gabanelli, and G. Remuzzi. 1991. Increased renal endothelin production in rats with reduced renal mass. Am. J. Physiol. 260:F331-F339.

16. Wesson, D.E., and G.M. Dolson. 1991. Augmented bidirectional $\mathrm{HCO}_{3}$ transport by rat distal tubules in chronic alkalosis. Am. J. Physiol. 261:F308F317.

17. Crayen, M.L., and W. Thoenes. 1978. Architecture and cell structures in the distal nephron of the rat kidney. Cytobiologie. 17:197-211.

18. Wesson, D.E. 1990. Depressed distal tubule acidification corrects chloride-deplete alkalosis in rats. Am J. Physiol. 259:F636-F644.

19. Star, R.A. 1990. Quantitation of total carbon dioxide in nanoliter samples by flow-through fluorometry. Am J. Physiol. 258:F429-F432.

20. Levine, D.Z., M. Iacovitti, L. Nash, and D. Vandorpe. 1988. Secretion of bicarbonate by rat distal tubules in vivo. Modulation by overnight fasting. $J$. Clin. Invest. 81:1873-1878.

21. Iacovitti, M., L. Nash, L.N. Peterson, J. Rochon, and D.Z. Levine. 1986. Distal tubule bicarbonate accumulation in vivo. Effect of flow and transtubular bicarbonate gradients. J. Clin. Invest. 78:1658-1665.

22. Capasso, G., R. Kinne, G. Malnic, and G. Giebisch. 1986. Renal bicarbonate reabsorption in the rat. I. Effects of hypokalemia and carbonic anhydrase. J. Clin. Invest. 78:1558-1567.

23. Chan, Y.L., G. Malnic, and G. Giebisch. 1989. Renal bicarbonate reabsorption in the rat. III. Distal tubule perfusion study of load dependence and bicarbonate permeability. J. Clin. Invest. 84:931-938.

24. Wesson, D.E. 1996. Dietary $\mathrm{HCO}_{3}$ reduces distal tubule acidification by increasing cellular $\mathrm{HCO}_{3}$ secretion. Am J. Physiol. 271:F132-F142.

25. Wesson, D.E., and G.M. Dolson. 1991. Maximal proton secretory rate of rat distal tubules is higher during chronic metabolic alkalosis. Am. J. Physiol. 261:F753-F759.

26. Levine, D., and H.R. Jacobson. 1986. The regulation of renal acid secretion: new observations from studies of distal nephron segments. Kidney Int. 29: 1099-1109.
27. Garcia-Austt, J., D.W. Good, M.B. Burg, and M.A. Knepper. 1985. Deoxycorticosterone-stimulated bicarbonate secretion in rabbit cortical collecting ducts: effects of luminal chloride removal and in vivo acid loading. Am. J Physiol. 249:F205-F212.

28. Knepper, M.A., D.W. Good, and M.B. Burg. 1985. Ammonia and bicarbonate transport by rat cortical collecting ducts perfused in vitro. Am. J. Physiol. 249:F870-F877.

29. Lucci, M.S., L.R. Pucacco, N.W. Carter, and T.D. DuBose, Jr. 1982. Evaluation of bicarbonate transport in the rat distal tubule: effects of acid-base status. Am. J. Physiol. 243:F335-F341.

30. Buerkert, J., D. Martin, and D. Trigg. 1983. Segmental analysis of the renal tubule in buffer production and net acid formation. Am. J. Physiol. 244 F442-F454.

31. Knepper, M.A., R. Packer, and D.W. Good. 1989. Ammonium transport in the kidney. Physiol. Rev. 69:179-248.

32. Breyer, M.D., J.P. Kokko, and H.R. Jacobson. 1986. Regulation of net bicarbonate transport in rabbit cortical collecting tubule by peritubular $\mathrm{pH}$, carbon dioxide tension, and bicarbonate concentration. J. Clin. Invest. 77:1650 1660.

33. McKinney, T.D., and K.K. Davidson. 1988. Effects of respiratory acidosis on $\mathrm{HCO}_{3}$ transport by rabbit collecting tubules. Am. J. Physiol. 255:F656F665.

34. Marsen, T.A., H. Schramek, and M.J. Dunn. 1994. Renal actions of endothelin. Linking cellular signaling pathways to kidney disease. Kidney Int. 45: 336-344.

35. Ling, B.N. 1995. Effect of luminal endothelin-1 on apical $\mathrm{Na}^{+}$and $\mathrm{Cl}^{-}$ channels in primary cultured rabbit CCT. Clin. Res. 43:47 $\alpha$. (Abstr.)

36. Tomita, K., H. Nonguchi, and F. Marumo. 1990. Effects of endothelin on peptide-dependent cyclic adenosine monophosphate accumulation along the nephron segments of the rat. J. Clin. Invest. 85:2014-2018.

37. Eiam-Ong, S., S.A. Hilden, A.J. King, C.A. Johns, and N.E. Madias 1992. Endothelin-1 stimulates the $\mathrm{Na}^{+} / \mathrm{H}^{+}$and $\mathrm{Na}^{+} / \mathrm{HCO}_{3}{ }^{-}$transporters in $\mathrm{rab}-$ bit renal cortex. Kidney Int. 42:18-24.

38. Wang, T.G., G. Malnic, G. Giebisch, and Y.L. Chan. 1993. Renal bicarbonate reabsorption in the rat. IV. Bicarbonate transport mechanisms in the early and late distal tubule. J. Clin. Invest. 91:2776-2883.

39. Emmons, C., and J.B. Stokes. 1994. Cellular actions of cAMP on $\mathrm{HCO}_{3}{ }^{-}$-secreting cells of rabbit CCD: dependence on in vivo acid-base status. Am. J. Physiol. 266:F528-F535.

40. Weinman, E.J., S. Shenolikar, and A.M. Kahn. 1987. cAMP-associated inhibition of $\mathrm{Na}^{+}-\mathrm{H}^{+}$exchanger in rabbit kidney brush border membranes. Am. J. Physiol. 252:F19-F25.

41. Garvin, J., and K. Sanders. 1991. Endothelin inhibits fluid and bicarbonate transport in part by reducing $\mathrm{Na}^{+} / \mathrm{K}^{+}$ATPase activity in the rat proximal straight tubule. J. Am. Soc. Nephrol. 2:976-982.

42. Neuster, D., S. Zaiss, and J.-P. Stasch. 1990. Endothelin receptors in cultured renal epithelial cells. Eur. J. Pharmacol. 176:241-243.

43. Terada, Y., K. Tomita, H. Nonoguchi, and F. Marumo. 1992. Different localization of two types of endothelin receptor mRNA in microdissected rat nephron segments using reverse transcription and polymerase chain reaction assay. J. Clin. Invest. 90:107-112.

44. Wilkes, B.M., M. Susin, P.F. Mento, C.M. Macica, E.P. Giraldi, E. Boss, and E.P. Nord. 1991. Localization of endothelin-like immunoreactivity in rat kidneys. Am J. Physiol. 260:F913-F920.

45. Kriz, W., and B. Kaissling. 1992. Structural organization of the mammalian kidney. In The Kidney. Physiology and Pathophysiology. D. Seldin and G. Giebisch, editors. Raven Press, New York. p. 207.

46. Uchida, S., F. Takemoto, E. Ogata, and K. Kuroka. 1992. Detection of endothelin-1 mRNA by RT-PCR in isolated rat renal tubules. Biochem. Biophys. Res. Commun. 188:108-113.

47. Veis, J.H., M.A. Dillingham, and T. Berl. 1990. Effects of prostacyclin on the cAMP system in cultured rat inner medullary collecting duct cells. Am. $J$ Physiol. 258:F1218-F1223.

48. Wesson, D.E. 1996. Prostacyclin increases distal tubule $\mathrm{HCO}_{3}$ secretion in the rat. Am J. Physiol. 271:F1183-F1192.

49. Korbmacher, C., E. Boulpaep, G. Giebisch, and J. Geibel. 1993. Endothelin increases $\left[\mathrm{Ca}^{2+}\right]_{i}$ in $\mathrm{M}-1$ mouse cortical collecting duct cells by a dual mechanism. Am. J. Physiol. 265:C349-C357.

50. Simonson, M.S., S. Wann, P. Mene, G.R. Dubyak, M. Kester, Y. Nakazato, J.R. Sedor, and M.J. Dunn. 1989. Endothelin stimulates phospholipase C, $\mathrm{Na}^{+} / \mathrm{K}^{+}$exchange, c-fos expression and mitogenesis in rat mesangial cells. $J$. Clin. Invest. 83:708-712.

51. Tsukahara, H., H. Ende, H.I. Magazine, W.F. Bahou, and M.S. Goligorsky. 1994. Molecular and functional characterization of the non-isopeptideselective ETB receptor in endothelial cells. Receptor coupling to nitric oxide synthase. J. Biol. Chem. 269:21778-21785. 